\title{
CONCERNING APPROACHABILITY OF SIMPLE CLOSED AND OPEN
}

\section{CURVES *}

\author{
BY \\ JOHN ROBERT KLINE
}

Schoenflies $\dagger$ was the first to formulate the converse of the fundamental theorem of C. Jordan $\ddagger$ that a simple closed curve§ lying wholly within a plane decomposes the plane into an inside and an outside region. The statement of this converse theorem is as follows: Suppose $K$ is a closed, bounded set of points lying in a plane $S$ and that $S-K=S_{1}+. S_{2}$, where $S_{1}$ and $S_{2}$ are point-sets such that (1) every two points of $S_{i}(i=1,2)$ can be joined by an arc lying entirely in $S_{i}(2)$ every arc joining a point of $S_{1}$ to a point of $S_{2}$ contains at least one point of $K(3)$ if $O$ is a point of $K$ and $P$ is a point not belonging to $K$, then $P$ can be joined to $O$ by an arc that has no point except $O$ in common with $K$. Every point-set that satisfies these conditions is a simple closed curve. Schoenflies used metrical hypotheses in his proof. Lennes gave a proof of this converse theorem using straight lines. $\|$ R. L. Moore pointed out that a proof similar in large part to that of Lennes can be carried through with the use of arcs and closed curves on the basis of his system of postulates $\Sigma_{3}$, thus furnishing a non-metrical proof of the converse theorem. 9

In all these proofs the condition numbered three, the condition of approachability (erreichbarkeit) plays a fundamental rôle. It is the purpose of the present paper to study the effect of substituting for the condition of approach-

* Presented to the Society, April, 1918.

† Cf. A. Schoenflies, Ueber einen grundlegenden Satz der Analysis Situs, N a c h r i c h t e $\mathbf{n}$ der Götinger Gesellschaft der Wissenschaften, 1902, p. 185.

$\ddagger$ C. Jordan, Cours d'Analyse, 2d ed., Paris, 1893, p: 92.

\& If $A$ and $B$ are distinct points, then a simple continuous arc from $A$ to $B$ is defined by Lennes as a bounded, closed, connected set of points containing $A$ and $B$, but containing no proper connected subset containing both $A$ and $B$, Cf. N. J. Lennes Curves in non-metrical analysis situs with an application in the calculus of variations, A m e r i c a $\mathbf{n}$ Jou $\mathbf{r} \mathbf{n}$ a of M a t h e m a ti c s, vol. 33 (1911), p. 308. A simple closed curve is a set of points composed of two arcs $A X B$ and $A Y B$ having no point in common other than $A$ and $B$. Hereafter in this paper "arc" and "closed curve" will be considered synonymous with "simple continuous arc" and "simple closed curve," respectively.

\| Cf. N. J. Lennes, loc. cit., $\$ 5$.

T Cf. R. L. Moore, On the foundations of plane analysis situs, these T ran s a c t i o $\mathrm{s}$, vol. 17 (1916), p. 59. 
ability, the condition that the set is "connected in kleinem."* The results obtained are embodied in the following theorem:

Theorem $A$. Suppose $K$ is a closed plane point-set, $S$ is the set of all points of the plane, while $S-K=S_{1}+S_{2}$, where $S_{1}$ and $S_{2}$ are two mutually exclusive domains $\dagger$ such that every point of $K$ is a common bu undary point of $S_{1}$ and $S_{2}$. Then a necessary and sufficient condition that $K$ be either a simple closed curve or an open curve $\ddagger$ is that $K$ be connected in kleinem.

That the condition stated in Theorem $A$ is necessary is evident. I will proceed to show that it is sufficient. Suppose $K$ is a connected in kleinem set satisfying the conditions stipulated in Theorem $A$. Then the following lemmas hold true:

Lemma $A$. Every arc joining a point of $S_{1}$ to a point of $S_{2}$ contains a point of $K$.

Proof. Suppose it were possible to draw an arc from a point $P_{1}$ of $S_{1}$ to a point $P_{2}$ of $S_{2}$ that contains no point of $K$. Then let us divide the arc $P_{1} P_{2}$ into two sets, $M_{1}$ and $M_{2}$, where $M_{1}$ is the set of all points of $P_{1} P_{2}$ that belong to $S_{1}$, while $M_{2}$ is the set of all points of $P_{1} P_{2}$ which belong to $S_{2}$. As $P_{1} P_{2}$ is a connected point-set either $M_{1}$ contains a limit point of $M_{2}$ or $M_{2}$ contains a limit point of $M_{1}$.

Case $I$. A point $F$ of $M_{1}$ is a limit point of $M_{2}$. As $F$ is a point of the domain $S_{1}$, there exists a region containing $F$ and lying entirely in $S_{1}$. As $S_{1}$ and $S_{2}$ are mutually exclusive domains, this region contains no point of $M_{2}$. Hence $F$ cannot be a limit point of $M_{2}$.

Case II. A point $G$ of $M_{2}$ is a limit point of $M_{1}$. This is impossible as in Case I.

Hence we are led to a contradiction if we suppose our lemma false.

LEmma $B$. The set $K$ is connected.

Proof. Suppose $K$ were not connected. Then it could be divided into two mutually exclusive sets $K_{1}$ and $K_{2}$, neither of which contains a limit point of the other one. Let $P_{i} \cdot(i=1,2)$ denote a point of $K_{i}$. Put about $P_{i}$ a circle $R_{i}$ having $P_{i}$ as center and such that $R_{i}$ and its interior lie entirely

* Cf. Hans Hahn, Ueber die allgemeinste ebene Punktmenge die stetiges Bild einer Strecke ist, Jahresbericht der Deutschen Mathematiker Vereinigung, vol. 23 (1914), pp. 318-22. According to Hahn, a set of points $C$ is said to be connected in kleinem if, whenever $P$ is a point of $C, \epsilon$ a positive number and $K$ a circle of radius $1 / \epsilon$ with center at $P$, then there exists within $K$ and with center at $P$, another circle $K_{e}, P$ such that if $X$ is a point of $C$ within $K_{\epsilon, P}$ then $X$ and $P$ lie together in some connected subset of $C$ that lies entirely within $K$.

$\dagger$ A domain is a connected set of points $M$ such that if $P$ is a point of $M$, then there is a region that contains $P$ and lies in $M$.

$\ddagger$ An open curve is defined by R. L. Moore as a closed, connected, set of points $M$ such that if $P$ is a point of $M$, then $M-P$ is the sum of two mutually exclusive connected point-sets, neither of which contains a limit point of the other. 
without $R_{i+1} .^{*} \quad$ As $K$ is connected in kleinem, there exists a circle $\bar{R}$, lying within $R_{i}$ and with center at $P_{i}$ such that if $X_{i}$ is a point of $K$ within $\bar{R}_{i}$, then $X_{i}$ and $P_{i}$ lie on some connected subset of $K$ lying within $R_{i}$. It may easily be shown that $X_{i}$ can be joined to $P_{i}$ by a simple continuous arc of $K$ lying entirely within $R_{i} . \dagger$ As every point of $K$ is a common boundary point of $S_{1}$ and $S_{2}$, then there exists within $\bar{R}_{i}$ a point $M_{i j}(j=1,2)$ belonging to $S_{j}$. As $S_{j}$ is a domain, then there exists a simple continuous arc $M_{1 j} K_{j} M_{2 j}$ lying entirely in $S_{j}$. Join $M_{i j}$ to $P_{i}$ by a simple continuous arc $M_{i j} L_{i j} P_{i}$ lying entirely within $\bar{R}_{i}$ and let $G_{i j}$ denote the first point of $K$ on the arc $M_{i j} L_{i j} P_{i}$ following $M_{i j}$. Then we may join $G_{i 1}$ to $G_{i 2}$ by an $\operatorname{arc} G_{i 1} F_{i} G_{i 2}$ belonging to $K$ and lying entirely within $R_{i}$. The point-set $G_{11} M_{11}$ (on $M_{11} L_{11} P_{1}$ ) $+M_{11} K_{1} M_{21}+M_{21} G_{21}$ (on $M_{21} L_{21} P_{2}$ ) contains as a subset a simple continuous arc $G_{11} H_{1} G_{21}$ lying except for its endpoints entirely in $S_{1}$, while the set $G_{12} M_{12}$ (on $\left.M_{12} L_{12} P_{1}\right)+M_{12} K_{2} M_{22}+M_{22} G_{22}$ (on $M_{22} L_{22} P_{2}$ ) contains as a subset a simple continuous arc $G_{12} H_{2} G_{22}$ lying except for its endpoints entirely in $S_{2}$. We then have a closed curve $G_{11} F_{1} G_{12} H_{2} G_{22}$ - $F_{2} G_{21} H_{1} G_{11}$ such that the arcs $G_{11} F_{1} G_{12}$ and $G_{21} F_{2} G_{22}$ lie entirely on $K$ and within $R_{1}$ and $R_{2}$, respectively, while $G_{11} H_{1} G_{21} \ddagger$ and $G_{12} H_{2} G_{22}$ belong to $S_{1}$ and $S_{2}$, respectively.

All points of $G_{11} F_{1} G_{12}$ belong to $K_{1}$. For suppose a point $H$ of $G_{11} F_{1} G_{12}$ belonged to $K_{2}$. As $H$ is joined to $G_{11}$, which in turn can be joined to $P_{1}$ by an arc of $K$ lying entirely within $R_{1}$, it follows that $H$ can be joined to $P_{1}$ by an arc $H F P_{1}$ of $K$ lying entirely within $R_{1}$. Let $\left[\bar{H}_{1}\right]$ denote the set of all points of $H F P_{1}$ belonging to $K_{1}$ while $\left[\bar{H}_{2}\right]$ denotes the set of all points of $H F P_{1}$ belonging to $K_{2}$. Clearly neither of these sets contains a limit point of the other. Hence the arc $H F P_{1}$ is not a connected point-set. Hence the supposition that $H$ belongs to $K_{2}$ has led to a contradiction. In like manner, all points of $G_{21} F_{2} G_{22}$ belong to $K_{2}$.

The interior of $G_{11} F_{1} G_{12} H_{2} G_{22} F_{2} G_{21} H_{1} G_{11}$ must contain at least one point of $K$. For suppose it does not contain a point of $K$. Then the interior of $G_{11} F_{1} G_{12} H_{2} G_{22} F_{2} G_{21} H_{1} G_{11}$ is a subset of $S_{1}+S_{2}$. Suppose it contains a point $H$ of $S_{1}$. Then $H$ can be joined to $H_{2}$ by an arc $H X H_{2}$ lying except for $H_{2}$ entirely within $G_{11} F_{1} G_{12} H_{2} G_{22} F_{2} G_{21} H_{1} G_{11} . \S$ Let [ $W_{1}$ ] denote the set of all points of $\mathrm{HXH}_{2}$ belonging to $S_{1}$ while [ $\dot{W}_{2}$ ] denotes the set of all points of $\mathrm{HXH}_{2}$ which are points of $S_{2}$. Clearly neither of these sets contains

* It is understood that subscripts are reduced modulo 2 ,

† Cf. R. L. Moore, A theorem concerning continuous curves, B ullet in of the A m e r i can Mathematical Society, vol. 23 (1917). While Professor Moore's theorem states that every two points of a continuous curve can be joined by a simple continuous arc lying entirely on the given continuous curve, it is clear that his methods suffice to prove the above stronger statement.

$\ddagger$ If $A X B$ is an arc, then the symbol $A X B$ will denote $A X B-A-B$,

$\$$ Cf. R. L. Moore, Foundations of plane analysis situs, loc. cit., Theorem 39, pp. 153-5. 
a limit point of the other. Hence the $\operatorname{arc} \mathrm{HXH}_{2}$ is not a connected point-set. In like manner the supposition that there is within $G_{11} F_{1} G_{12} H_{2} G_{22} F_{2}$ $G_{21} H_{1} G_{11}$, a point of $S_{2}$ leads to a contradiction. Hence $G_{11} F_{1} G_{12} H_{2} G_{22} F_{2}$ $G_{21} H_{1} G_{11}$ must enclose a point of $K$.

Let [ $V_{2}$ ] denote the set of all points $V_{2}$ such that either (1) $V_{2}$ is a point of $G_{21} F_{2} G_{22}$, or (2) $V_{2}$ is a point such that there exists a closed connected set $V_{2} X F_{2}^{\prime}$ belonging to $K$ and lying within or on $G_{11} F_{1} G_{12} H_{2} G_{22} F_{2} G_{21} H_{1} G_{11}$. and such that $F_{2}^{\prime}$ is a point of $G_{21} F_{2} G_{22}$. As $K$ is connected in kleinem it may easily be proved that $\left[V_{2}\right]$ is a closed set. It is also true that all points of $\left[V_{2}\right]$ belong to $K_{2}$. Hence no point of $G_{11} F_{1} G_{12}$ either belongs to or is a limit point of $\left[V_{2}\right]$. It may also be proved with the use of the in kleinem property that no point of $\left[V_{2}\right]$ is a limit point of a set of points of $K$ lying within $G_{11} F_{1} G_{12} H_{2} G_{22} F_{2} G_{21} H_{1} G_{11}$ and containing no point of $V_{2}$. There exists an arc $H_{1} Y H_{2}$ such that (1) $H_{1} Y H_{2}$ is a subset of the interior of $G_{11} F_{1} G_{12} H_{2} G_{22} F_{2} G_{21} H_{1} G_{11}$ and (2) $\bar{H}_{1} Y H_{2}$ contains no points of [ $\left.V_{2}\right]^{*}{ }^{*}$ Let $\left[V_{1}\right]$ denote the set of all points of $K$ within or on the closed curve, $H_{1} Y H_{2} G_{22} F_{2} G_{21} H_{1}$, not belonging to [ $\left.V_{2}\right]$. The set [ $V_{1}$ ] is closed. Put about each point of [ $\left.V_{1}\right]$ a circle lying entirely within $G_{11} F_{1} G_{12} H_{2} G_{22} F_{2}$ $G_{21} H_{1} G_{11}$ and containing within it or on its boundary no point of [ $\left.V_{2}\right]$. By the Heine-Borel Property, there exists a finite number of circles of the above set, $C_{1}, C_{2}, \cdots, C_{n}$, covering [ $\left.V_{1}\right]$. With the use of Theorems $41,42,43$, and 44 of Professor Moore's Foundations we may easily obtain from the set $C_{1}, C_{2}, \cdots, C_{n}$ and the closed curve $G_{11} F_{1} G_{12} H_{2} Y H_{1} G_{11}$, a new closed curve $G_{11} F_{1} G_{12} H_{2} Z H_{1} G_{11}$, where the $\operatorname{arc} H_{1} G_{11} F_{1} G_{12} H_{2}$ of the new closed curve $G_{11} F_{1} G_{12} H_{2} Z H_{1} G_{11}$ is the arc $H_{1} G_{11} F_{1} G_{12} H_{2}$ of $G_{11} F_{1} G_{12} H_{2} Y H_{1} G_{11}$ and where $H_{2} Z H_{1}$ is free from points of $K$ and lies within $G_{11} F_{1} G_{12} H_{2} G_{22} F_{2}$ $G_{21} H_{1} G_{11}$. But then we have a point of $S_{1}$ joined to a point of $S_{2}$ by an arc containing no point of $K$. Thus the supposition that $K$ is not connected, leads to a contradiction.

Lemma $C$. If $K$ contains one simple closed curve $J$, then all points of $K$ belong to $J$.

Proof. Suppose Lemma $C$ is not true. Then $K$ contains a closed curve $J$ and at least one point $P$ not on $J$. Two cases may arise:

Case $I$. $P$ is within $J$. As every point of $K$ is a common boundary point of $S_{1}$ and $S_{2}$, the interior of $J$ contains a point $P_{1}$ of $S_{1}$ and a point $P_{2}$ of $S_{2}$. The exterior of $J$ cannot contain a point $\bar{P}_{1}$ of $S_{1}$. For suppose it did. Then any arc from $P_{1}$ to $\bar{P}_{1}$ would contain a point of $J$ and hence a point of $K$, contrary to the fact that $S_{1}$ is a domain. In like manner no point of $S_{2}$ can be in the exterior of $J$. Hence the exterior of $J$ must be a subset of $K$, while

* Cf. my paper, $A$ definition of sense on plane closed curves in non-metrical analysis situs, Annals of Mathematics, vol. XIX (1918), Theorem D, pp. 188-9. 
$S_{1}$ and $S_{2}$ are subsets of the interior of $J$. But this is impossible because no point without $J$ is a limit point of a set of points lying entirely within $J$ thus making it impossible that every point of $K$ be a common boundary point of $S_{1}$ and $S_{2}$. Hence the supposition that $P$ is within $J$ has led to a contradiction.

Case $I I$. $P$ is without $J$. Case II may be proved impossible by an argument similar to that used in Case I.

An immediate consequence of Lemma $C$ is that if $K$ is not a simple closed curve, then there is but one $K$-arc from a point $A$ of $K$ to a distinct point $B$ of $K$.

Lemma $D$. The set $K$ does not contain three arcs $O P_{1}, O P_{2}$, and $O P_{3}$, no two of which have a common point other than $O$.

Proof. Suppose Lemma $D$ were false. Then there would exist three arcs $O P_{1}, O P_{2}$, and $O P_{3}$, no two of which have a point in common other than $O$. Put about $P_{i}(i=1,2,3)$ a circle $C_{i}$ such that the point-set $O P_{i+1}{ }^{*}+O P_{i+2}$ is a subset of the exterior of $C_{i}$ and such that $C_{i}$ has no point in common with $C_{i+1}+C_{i+2}$. As $K$ is connected in kleinem, there exists within $C_{i}$ and with center at $P_{i}$, another circle $C_{P_{i}}, C_{i}$ such that if $X_{i}$ is a point of $K$ within $C_{P_{i}}, C_{i}$, then there is an arc from $X_{i}$ to $P_{i}$ every point of which is a point of $K$ and which lies entirely within $C_{i}$. As all points of $K$ are limit points of both $S_{1}$ and $S_{2}, C_{P_{i}, C_{i}}$ must contain at least one point $P_{i, 1}$ of $S_{1}$. As $S_{1}$ is a domain, there is an arc $P_{11} P_{21}$ from $P_{11}$ to $P_{21}$ all points of which belong to $S_{1}$. Join $P_{i, 1}$ to $P_{i}$ by an arc $P_{i, 1} P_{i}$ lying entirely within $C_{P_{i}, C_{i}}$ and let $X_{i}$ denote the first point of the $\operatorname{arc} P_{i, 1} P_{i}$ after $P_{i, 1}$, which belongs to $K$. There exists an $\operatorname{arc} X_{i} P_{i}$ from $X_{i}$ to $P_{i}$ belonging to $K$ and lying entirely within $C_{i}$. Let $P_{i}^{\prime}$ denote the first point of the $\operatorname{arc} X_{i} P_{i}$ which is on $O P_{i}$. The point-set $P_{1}^{\prime} X_{1}+X_{1} P_{11}+P_{11} P_{21}+P_{21} X_{2}+X_{2} P_{2}^{\prime}$ contains as a subset an arc $P_{1}^{\prime} F_{1} P_{2}^{\prime}$ such that (1) $P_{1}^{\prime} F_{1} P_{2}^{\prime}$ has no point in common with $O P_{1}+O P_{2}$ $+O P_{3}$, (2) all points of $P_{1}^{\prime} F_{1} P_{2}^{\prime}$ belong to either $K$ or $S_{1}$, (3) at least one point, $F_{1}$, of $S_{1}$ is a point of $P_{1}^{\prime} F_{1} P_{2}^{\prime}$. By methods similar to those just employed, we may construct an arc $Q_{1}^{\prime} H_{2} Q_{2}^{\prime}$ from a point $Q_{1}^{\prime}$ of $O P_{1}$ to a point $Q_{2}^{\prime}$ of $O P_{2}^{\prime}$ such that (1) $Q_{i}^{\prime}$ is on $O P_{i}$ between $P_{i}^{\prime}$ and $O$, (2) all points of $Q_{1}^{\prime} H_{2} Q_{2}^{\prime}$ belong to either $S_{2}$ or $K$, (3) except for $Q_{1}^{\prime}$ and $Q_{2}^{\prime}, Q_{1}^{\prime} H_{2} Q_{2}^{\prime}$ has no point in common with $P_{1}^{\prime} F P_{2}^{\prime}+O P_{1}+O P_{2}+O P_{3}$, (4) at least one point $H_{2}$ of $Q_{1}^{\prime} H_{2} Q_{2}^{\prime}$ belongs to $S_{2}$. Two cases may arise:

Case I. $Q_{1}^{\prime} H_{2} Q_{2}^{\prime}$ is entirely within $O P_{1}^{\prime} F_{1} P_{2}^{\prime} O$. Then the interior of $O P_{1}^{\prime} F_{1} P_{2}^{\prime} O=Q_{1}^{\prime} H_{2} Q_{2}^{\prime}+$ the interior of $O Q_{1}^{\prime} H_{2} Q_{2}^{\prime} O+$ the interior of

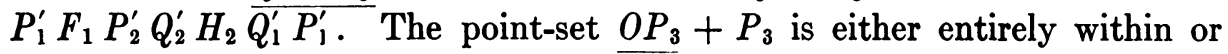
entirely without $O Q_{1}^{\prime} H_{2} Q_{2}^{\prime} O$.

(a) Suppose $O P_{3}+P_{3}$ is entirely within $O Q_{1}^{\prime} H_{2} Q_{2}^{\prime} O$. Then $O Q_{1}^{\prime} H_{2} Q_{2}^{\prime} O$

* It is understood throughout this argument that subscripts are reduced modulo 3.

t See an earlier footnote. 
must enclose at least one point $L$ of $S_{1}$. But then an arc from $L$ to $F_{1}$ must contain at least one point of $O Q_{1}^{\prime} H_{2} Q_{2}^{\prime} O$. Hence, as $O Q_{1}^{\prime} H_{2} Q_{2}^{\prime} O$ is a subset of $K+S_{2}$, no such arc $L F_{1}$ can lie entirely in $S_{1}$, contrary to the fact that $S_{1}$ is a domain.

(b) Suppose $O P_{3}+P_{3}$ is entirely without $O Q_{1}^{\prime} H_{2} Q_{2}^{\prime} O$. It follows that $O P_{3}+P_{3}$ is entirely without $O P_{1}^{\prime} F_{1} P_{2}^{\prime} O$. Then the exterior of $O P_{1}^{\prime} F_{1} P_{2}^{\prime} O$ contains at least one point $M$ of $S_{2}$. Then any arc from $M$ to $H_{2}$ must contain at least one point of $O P_{1}^{\prime} F_{1} P_{2}^{\prime} O$ and hence at least one point not in $S_{2}$. But this is contrary to the fact that $S_{2}$ is a domain.

Thus in Case I we are led to a contradiction.

Case II. $Q_{1}^{\prime} H_{2} Q_{2}^{\prime}$ is without $O P_{1}^{\prime} F_{1} P_{2}^{\prime} O$. We may show that Case II is impossible by methods similar to those used in Case I.

Lemma $E$. If $O$ is a point of $K$ and $P$ is a point of $S_{i}(i=1,2)$ then there exists at least one arc $O P$ such that $O P+P$ is a subset of $S_{i}$.

Proof. Two conceivable cases may arise.

Case I. There exist points $A_{1}$ and $A_{2}$ of $K\left\lfloor A_{1} \neq O \neq A_{2}\right]$ such that $O$ is a point of the arc $A_{1} O A_{2}$ belonging to $K$. By the same methods as were used in the preceding lemma we may construct an arc $A_{1}^{\prime} F_{1} A_{2}^{\prime}$ such that (1) on $A_{1} O A_{2}$ the order $A_{1} A_{1}^{\prime} O A_{2}^{\prime} A_{2}$ holds, (2) $A_{1}^{\prime} F_{1} A_{2}^{\prime}$ is a subset of $S_{1}+K$, (3) at least one point $F_{1}$ of $A_{1}^{\prime} F_{1} A_{2}^{\prime}$ is a point of $S_{1}$, (4) no point of $A_{1}^{\prime} F A_{2}^{\prime}$ belongs to $A_{1} O A_{2}$. The point $O$ is not a limit point of $K-A_{1}^{\prime} O A_{2}^{\prime}$. For suppose it were. Then it would be a sequential limit point of a set of points $P_{1}, P_{2}, \cdots$, every one of which belongs to $K-A_{1}^{\prime} O A_{2}^{\prime}$. Put about $O$ as center a circle $M$ such that $A_{1}^{\prime}$ and $A_{2}^{\prime}$ are both without $M$. As $K$ is connected in kleinem there exists another circle $\bar{M}$ lying within $M$ and having its center at $O$ such that if $X$ is a point of $K$ within $\bar{M}$, then $X$ and $O$ can be joined by an arc of $K$ lying entirely within $M$. Let $\bar{P}$ denote that point of the set $P_{1}, P_{2}, \cdots$ of lowest subscript which lies within $\bar{M}$, while $\bar{P} O$ denotes an arc of $K$ from $\bar{P}$ to $O$ lying entirely within $M$. Let $O^{\prime}$ denote the first point of $\bar{P} O$ which is on $A_{1}^{\prime} O A_{2}^{\prime}$. Then the set $K$ contains three $\operatorname{arcs} A_{1}^{\prime} O^{\prime}, A_{2}^{\prime} O^{\prime}$, and $P O^{\prime}$, no two of which have a point in common other than $O^{\prime}$. But this is contrary to Lemma $D$. Hence $O$ cannot be a limit point of $K-A_{1}^{\prime} O A_{2}^{\prime}$. There exists a closed curve $G$ enclosing $O$ but enclosing no points of $A_{1}^{\prime} F A_{2}^{\prime}$ $+\left[K-A_{1}^{\prime} O A_{2}^{\prime}\right]$. Then there exist two closed curves $J_{1}^{\prime}$ and $J_{2}^{\prime}$ such that (1) every point of $J_{1}^{\prime}$ or $J_{2}^{\prime}$ belongs either to $G$ or to $A_{1}^{\prime} F_{1} A_{2}^{\prime} O A_{1}^{\prime}(2) O$ is on $J_{1}^{\prime}$ and on $J_{2}^{\prime}(3)$ every point within $J_{1}^{\prime}$ is within $A_{1}^{\prime} F_{1} A_{2}^{\prime} O A_{1}^{\prime}$ while every point within $J_{2}^{\prime}$ is without $A_{1}^{\prime} F_{1} A_{2}^{\prime} O A_{1}^{\prime}(4)$ every point within either $J_{1}^{\prime}$ or $J_{2}^{\prime}$ is within $G .^{*}$ It is clear that either the interior of $J_{1}^{\prime}$ or the interior of $J_{2}^{\prime}$ is a subset of $S_{1}$ while the interior of the other of these two closed curves is a subset of $S_{2}$. Let $J_{1}$ denote that one whose interior is a subset of $S_{1}$ while

* Cf. R. L. Moore, Foundations, Theorem 43, pp. 156-7. 
$J_{2}$ denotes the one whose interior is a subset of $S_{2}$. Let $E$ denote a point within $J_{1}$, while $P_{1}$ is any other point of $S_{1}$. There exists an arc $E O$ such that $E O-O$ is a subset of the interior of $J_{1}{ }^{*}$ As $S_{1}$ is a domain, there is an arc $E P_{1}$ lying entirely in $S_{1}$. The point-set $E O+E P_{1}$ contains as a subset an arc from $P_{1}$ to $O$ lying except for $O$ entirely in $S_{1}$. In like manner we may show that any point $P_{2}$ of $S_{2}$ can be joined to $O$ by an arc lying except for $O$ entirely in $S_{2}$.

Case II. There do not exist two distinct points $A_{1}$ and $A_{2}$ of $K$ such that $O$ is on an arc of $K$ from $A_{1}$ to $A_{2}$. Let $A$ denote a point of $K$ different from $O$ while $A R O$ denotes an arc of $K$ from $A$ to $O$. By an argument similar to that employed in Case I we may show that if $O$ were a limit point of $K$ - $A R O$, then either there would exist three arcs $A R^{\prime}, R^{\prime} O$, and $R^{\prime} P$, no two of which have a point in common other than $R^{\prime}$ or there would exist a point $A^{\prime},\left(A \neq A^{\prime} \neq O\right)$ such that $O$ is an arc of $K$ from $A^{\prime}$ to $A$. But the first of these possibilities contradicts Lemma $D$ while the second is contrary to the hypothesis of Case II. Hence $O$ cannot be a limit point of $K-A R O$. Put about $O$ a circle $C$ that neither contains or encloses any point of $K-A R O$. Let $P_{1}, P_{2}, \cdots$ denote a set of points of $S_{1}$ approaching $O$ as their sequential limit point. It is possible to pass at least one simple continuous arc $\dagger$ through $A R O+P_{1}+P_{2}+\cdots$. Let $P_{1}^{\prime} O R A$ denote one such arc. If the interval $O P_{1}^{\prime}$ of the arc $P_{1}^{\prime} O R A$ does not lie entirely with in $C$, let $P^{\prime}$ denote the first point which it has in common with $C$. Otherwise let $P^{\prime}$ denote $P_{1}^{\prime}$. Let $\bar{P}$ denote that point of the set $P_{1}, P_{2}, \cdots$ of lowest subscript lying on $O P^{\prime}$. It is clear that the sub-arc $O \bar{P}$ of $P_{1}^{\prime} O R A$ lies, except for $O$, entirely in $\overline{S_{1}}$. Let $F_{1}$ denote any other point of $S_{1}$. Join $F_{1}$ to $\bar{P}$ by an arc lying entirely in $S_{1}$. Then the point-set $O \bar{P}+\bar{P} F_{1}$ contains as a subset an arc from $O$ to $F_{1}$, lying except for $O$ entirely in $S_{1}$.

In like manner we may show that if $F_{2}$ is a point of $S_{2}, F_{2}$ can be joined to $O$ by an arc lying except for $O$ entirely in $S_{2}$.

Lemma $F . \quad A$ necessary and sufficient condition that $K$ be bounded, is that either $S_{1}$ or $S_{2}$ be bounded.

Proof. The condition is necessary. Let us suppose that $K$ is bounded while neither $S_{1}$ nor $S_{2}$ is bounded. As $K$ is bounded, there is a circle $C$ such that all points of $K$ are within $C$. As $S_{1}$ and $S_{2}$ are unbounded, there is a point $P_{1}$ of $S_{1}$ and a point $P_{2}$ of $S_{2}$ without $C$. Join $P_{1}$ and $P_{2}$ by an arc lying entirely without $C$. By Lemma $A$, this arc must contain a point of $K$. But all points of $K$ are within $C$. Hence we are led to a contradiction if we suppose our condition is not necessary.

${ }^{*}$ Cf. R. L. Moore, Foundations, Theorem 39, pp. 153-5.

† Cf. R. L. Moore and J. R. Kline, On the most general closed point-set through which it is possible to pass a simple continuous arc, A $\mathrm{n} n$ a l s of $\mathrm{M}$ a t h e $\mathrm{m}$ a t i c s, vol. XX (1919), pp. 218-23. 
The condition is sufficient. For suppose $S_{1}$ is bounded while $K$ is unbounded. Since $S_{1}$ is bounded, there exists a circle $C$ enclosing $S_{1}$. Since $K$ is unbounded, it contains a point $P$ without $C$. The point $P$ cannot be a limit point of $S_{1}$. But this is contrary to hypothesis.

Proof of Theorem $A$. Two cases may arise:

Case I. $K$ is bounded. Then, by Schoenflies' Theorem and the preceding lemmas, it follows that $K$ is a simple closed curve.

Case II. $K$ is unbounded. It follows, by Lemma $F$ that neither $S_{1}$ nor $S_{2}$ is bounded. Then $K$ is an open curve. For a proof of this statement see my paper, "The converse of the theorem concerning the division of a plane by an open curve."*

University of Pennsyluania, Philadelphia, Pa.

*Cf. these Transaction s, vol. 18 (1917), pp. 177-184. 